\title{
EFECTO DEL SISTEMA DE ESTERILIZACIÓN ORGÁNICA (OSS) EN LAS PROPIEDADES NUTRICIONALES Y FUNCIONALES DEL YACÓN (Smallantus sonchifolius Poepp. \& Endl.)
}

\author{
Américo Guevara Pérez ${ }^{\mathrm{a}}$, Keidy Cancino Chávez ${ }^{\mathrm{b}}$, Gabriela Llontop ${ }^{\mathrm{c}}$
}

\begin{abstract}
RESUMEN
La máxima conservación química y funcional del yacón, por el sistema de esterilización orgánica, se logró aplicando una carga de $4 \mathrm{~kg} /$ saquillo, 1 minuto de tiempo de proceso y $100{ }^{\circ} \mathrm{C}$ temperatura de inyección de vapor saturado. Bajo estas condiciones se obtuvo $2,4 \%$ (b.s.) de proteínas y $52 \%$ (b.s.) de fructooligosacáridos. Respecto a la carga microbiana, se redujo 60,59 \% (10 UFC/g), 65,76 \% (20 UFC/g) y $0 \%(<10 \mathrm{UFC} / \mathrm{g})$ para aerobios, mohos y levaduras, respectivamente; el comportamiento de patógenos fue muy similar al de la materia prima: E. coli $<3 \mathrm{UFC} / \mathrm{g}$, ausencia de salmonella $(25 \mathrm{~g})$ y $<3 \mathrm{UFC} / \mathrm{g}$ para coliformes totales, cuyas muestras se lograron ajustar a las exigencias de la norma sanitaria peruana y a las normas internacionales; lo que evidenció que al aplicar la tecnología OSS se logra conservar la calidad nutricional y funcional del yacón.
\end{abstract}

Palabras clave: Smallantus sonchifolius, yacón, sistema de esterilización orgánica (OSS).

\section{ORGANIC STERILIZATION SYSTEM (OSS) EFFECT IN NUTRITIONAL AND FUNCIONAL PROPERTIES OF YACON (Smallantus sonchifolius Poepp. \& Endl.)}

\begin{abstract}
The maximum chemical and functional conservation of the yacón by the organic sterilization system, was achieved by applying a load of $4 \mathrm{~kg} /$ pouch, $1 \mathrm{~min}$. process time and temperature $100{ }^{\circ} \mathrm{C}$ saturated steam injection. Under these conditions, was obtained $2,4 \%(\mathrm{db})$ protein and $52 \%(\mathrm{db})$ of fructooligosaccharides. Regarding the microbial load, 60,59\% (10 CFU / g), $65,76 \%(20 \mathrm{CFU} / \mathrm{g})$ and $0 \%(<10 \mathrm{CFU} / \mathrm{g})$ for aerobes, molds, and yeasts, respectively decreased; pathogenic behavior was very similar to that of the raw material: E. coli $<3 \mathrm{CFU} /$ $\mathrm{g}$, absence of Salmonella ( $25 \mathrm{~g}$ ) and $<3 \mathrm{CFU} / \mathrm{g}$ for total coliforms, whose samples were able to adjust to the demands of the health standard Peruvian and international standards; which

\footnotetext{
${ }^{a}$ Universidad Nacional Agraria La Molina. Facultad de Industrias Alimentarias, Av. La Molina s/n, Ap. 12-056

Lima 12, Perú. aguevara@lamolina.edu.pe

b Universidad San Ignacio de Loyola. Facultad de ingeniería. kdcancino@yahoo.com

c Peruvian Nature S\&S SAC. gllontop@peruviannature.com
} 
showed that applying the OSS technology is accomplished to preserve the nutritional and functional quality of yacon.

Key words: Smallantus sonchifolius, Yacón, organic sterilization system (OSS).

\section{INTRODUCCIÓN}

Los cultivos andinos, como los granos, tubérculos, raíces, frutas, plantas aromáticas y medicinales, tienen gran potencial de transformación en productos procesados. El yacón (Smallanthus sonchifolius [Poepp. \&Endl.] H. Robinson) es una raíz nativa que no requiere de fertilizantes químicos ni pesticidas, con potencial para personas que sufren de diabetes, dado a que tanto en las raíces como en las hojas han encontrado una acción hipoglucemiante, antioxidante, con propiedades prebióticas y antienvejecimiento (raíces) ${ }^{1,2}$.

El mercado para el yacón y sus derivados es muy prometedor, si a ello sumamos otros atributos como la certificación orgánica, el comercio justo y el desarrollo de alternativas modernas, amigables y confiables de desinfección, pasteurización o esterilización, aumentaría significativamente.

Las tecnologías de desinfección existentes (irradiación, óxido de etileno, ozono y UV) están prohibidas en algunos países, entre ellos Japón, que aunado a las nuevas exigencias de inocuidad de alimentos donde se demanda ciertos niveles microbiológicos y a la vez restringen la aplicación de algunos métodos de esterilización convencional ${ }^{5}$; hacen que el Sistema de Esterilización Orgánica "Organic Sterilization System (OSS)", basada en someter al producto a cambios de presión y temperatura dentro de una cámara de vacío por un tiempo determinado, representa una solución natural, eficiente, reconocida y permitida internacionalmente, para lograr la conservación de las propiedades químicas y funcionales, así como la descontaminación microbiana del producto ${ }^{3}$.

Por lo expuesto, se llevó a cabo la investigación con el objetivo de determinar los parámetros del proceso OSS de descontaminación del yacón para preservar sus propiedades nutricionales y sensoriales.

\section{PARTE EXPERIMENTAL}

\section{Lugar de ejecución}

La investigación se desarrolló en instalaciones de la empresa Peruvian Nature S\&S S.A.C, y en los laboratorios del Instituto de Certificación, Inspección y Ensayo La Molina Calidad Total Laboratorios, pertenecientes a la Universidad Nacional Agraria La Molina.

\section{Análisis realizados}

Análisis físico-químico. Químico proximal4: Humedad, proteínas, grasa, ceniza, fibra, carbohidratos (por diferencia) y energía (calorías) y fructooligosacáridos ${ }^{5}$. 
Análisis microbiológico ${ }^{6}$. Aerobios mesófilos viables (UFC/g), levaduras (UFC/g), mohos (UFC/g). Salmonella sp. (25g), coliformes totales (NMP/g) y E. coli (NMP/g),

\section{Metodología experimental}

Acondicionamiento del yacón (Tratamiento PRE-OSS): Las raíces de yacón orgánico adquiridas en el Mercado de Productores de Santa Anita-Lima, fueron seleccionadas y sometidas a análisis de E. coli, salmonella y coliformes; luego se lavó, desinfectó, cortó en trozos de 10x10x10 mm y secó a $60{ }^{\circ} \mathrm{C}$. En esta etapa se evaluó: aerobios mesófilos viables, mohos y levaduras, tal como se puede apreciar en la tabla 1.

Posteriormente, los trozos deshidratados fueron acondicionados en saquillos de yute de 3 y $4 \mathrm{~kg}$ y puestos en bandejas para ser transportados en coches de carga a la cámara de esterilización. Los trozos deshidratados fueron analizados en: proximal y contenido de fructooligosacáridos.

Tabla 1. Diseño factorial del proceso de descontaminación microbiana por el sistema de esterilización orgánica (Tratamiento OSS).

\begin{tabular}{cccc}
\hline $\begin{array}{c}\text { Puntos } \\
\text { experimentales }\end{array}$ & $\begin{array}{c}\text { Carga } \\
(\mathbf{k g} / \mathbf{s a q u i l l o})\end{array}$ & $\begin{array}{c}\text { Tiempo de } \\
\text { proceso } \\
(\mathbf{m i n})\end{array}$ & $\begin{array}{c}\text { Temperatura de } \\
\text { inyección vapor } \\
\left({ }^{\circ} \mathbf{C}\right)\end{array}$ \\
\hline T1 & 3 & 1 & 100 \\
T2 & 4 & 1 & 100 \\
T3 & 3 & 2 & 100 \\
T4 & 4 & 2 & 100 \\
T5 & 3 & 1 & 105 \\
T6 & 4 & 1 & 105 \\
T7 & 3 & 2 & 105 \\
T8 & 4 & 2 & 105 \\
\hline
\end{tabular}

Descontaminación microbiana por el sistema de esterilización orgánica (Tratamiento OSS): El producto acondicionado, previamente en saquillos de 3 y $4 \mathrm{~kg}$ para $160 \mathrm{~kg} / \mathrm{Bach}$, ingresó a la cámara de proceso para su desinfección. El tratamiento OSS se llevó a cabo en 4 etapas: a) Preparación: Se evacuó el aire de la cámara de vacío preparándola para la inyección de vapor saturado. b) Tratamiento OSS: Se inyectó vapor saturado a temperaturas programadas de 100 y $105^{\circ} \mathrm{C}$ por tiempos de 1 y 2 minutos (tabla 1), con la finalidad de evitar la penetración excesiva del vapor y humedad al interior del producto. c) Evacuado de la cámara de vacío, se realizó extrayendo eficientemente el vapor inyectado. d) Secado a $60{ }^{\circ} \mathrm{C}$. Ejecutado el tratamiento OSS, las muestras fueron secadas en condiciones asépticas, hasta una humedad de 4-5\% cuya finalidad fue eliminar el agua remanente y de este modo lograr su estabilidad. En el producto estabilizado se realizó los siguientes análisis: Aerobios mesófilos viables, mohos y levaduras, E. coli, salmonella y coliformes totales. 
La molienda se llevó a cabo en un molino de martillo (aprox. 80 mesh). La harina fue directamente recibida en el área de envasado, donde a través de mangas ingresó a la envasadora automática.

En la figura 1 se muestra el flujo de operaciones del proceso de descontaminación microbiana del yacón por el sistema de esterilización orgánica (tratamiento OSS).

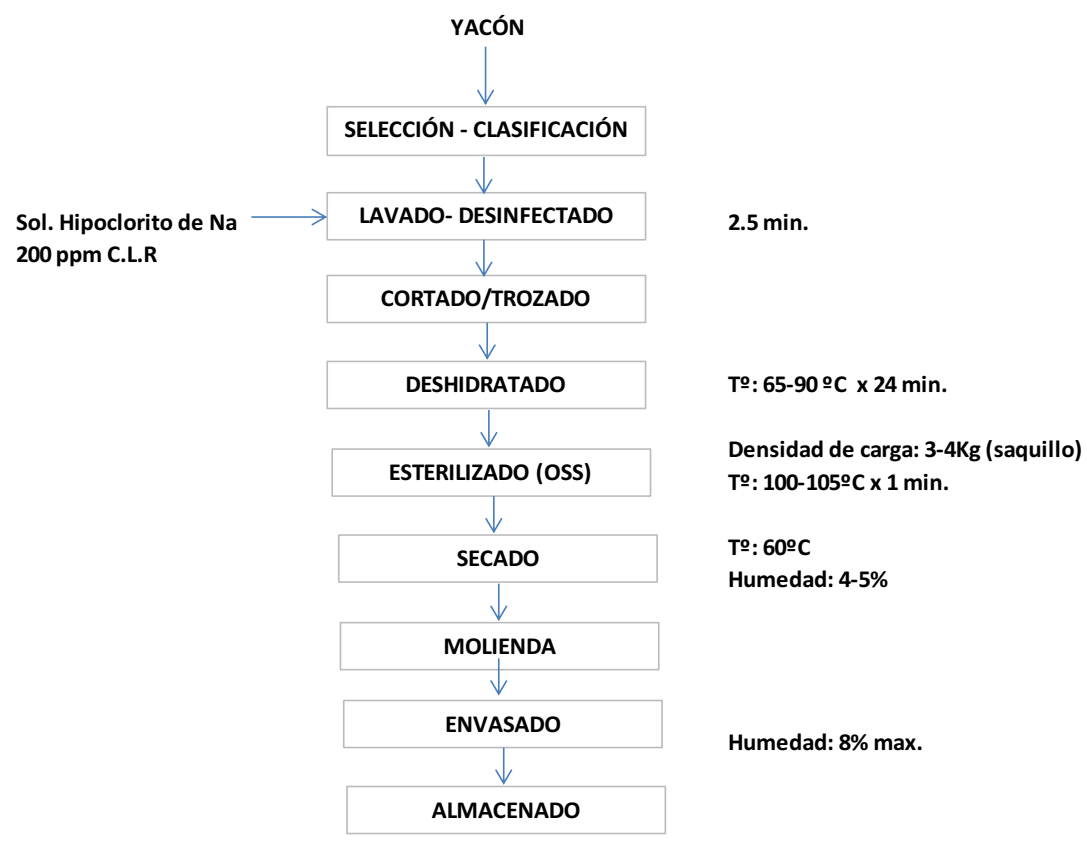

Figura 1. Flujo de operaciones del proceso de descontaminación microbiana del yacón por el sistema de esterilización orgánica (Tratamiento OSS).

\section{Caracterización del producto final}

Para evaluar la calidad de la harina de yacón (mejor tratamiento: máxima descontaminación) se realizó los siguientes análisis: proximal, fructooligosacáridos y carga microbiana: aerobios mesófilos viables, mohos y levaduras, E. coli, salmonella y coliformes totales.

\section{Diseño experimental y análisis estadístico}

Se aplicó un diseño factorial $2^{3}$ (8 puntos factoriales) para evaluar el efecto de la carga/ saquito ( 3 y $4 \mathrm{~kg}$ ), tiempo de proceso ( 1 y 2 minutos) y temperatura de inyección de vapor saturado $\left(100\right.$ y $\left.105^{\circ} \mathrm{C}\right)$ sobre la carga microbiana (aerobios mesófilos viables, levaduras y mohos), con el propósito de determinar los parámetros del proceso de descontaminación microbiana del yacón por el sistema de esterilización orgánica (Tratamiento OSS), para preservar sus propiedades nutricionales y funcionales. El programa estadístico utilizado fue el Statgraphics ${ }^{\circledR}$ Centurion XVI. 


\section{RESULTADOS Y DISCUSIÓN}

\section{Acondicionamiento del yacón (Tratamiento PRE-OSS)}

En la tabla 2 se presentan los resultados de la carga microbiana del yacón al Tratamiento PRE-OSS, reportando en la materia prima $<3(\mathrm{NMP} / \mathrm{g})$ para $E$. coli, coliformes totales y ausencia de salmonella.

Tabla 2. Comportamiento de la carga microbiana del yacón al tratamiento PRE-OSS.

\begin{tabular}{cccccccc}
\hline \multirow{2}{*}{ Tratamiento } & \multirow{2}{*}{$\begin{array}{c}\text { Momento del } \\
\text { análisis }\end{array}$} & \multicolumn{3}{c}{ Carga microbiana } & \multicolumn{3}{c}{ Patógenos } \\
\cline { 3 - 7 } & $\begin{array}{c}\text { Aerobios } \\
\text { (UFC/g) }\end{array}$ & $\begin{array}{c}\text { Mohos } \\
\text { (UFC/g) }\end{array}$ & $\begin{array}{c}\text { Levaduras } \\
(\mathrm{UFC} / \mathrm{g})\end{array}$ & $\begin{array}{c}\text { E. coli } \\
(\mathrm{NMP} / \mathrm{g})\end{array}$ & $\begin{array}{c}\text { Salmonella } \\
\text { (UFC/g) }\end{array}$ & $\begin{array}{c}\text { Coliformes } \\
(\mathrm{NMP} / \mathrm{g})\end{array}$ \\
\hline Materia prima & Yacón raíz & & & & $<3$ & ausencia & $<3$ \\
T1 & PRE OSS & 2600 & 50 & $<10$ & & \\
T2 & PRE OSS & 2000 & 110 & $<10$ & & \\
T3 & PRE OSS & 12000 & $<10$ & $<10$ & & \\
T4 & PRE OSS & 5100 & 50 & $<10$ & & \\
T5 & PRE OSS & 3800 & 70 & 10 & & \\
T6 & PRE OSS & 5100 & 10 & $<10$ & & \\
T7 & PRE OSS & 10000 & 20 & $<10$ & & \\
T8 & PRE OSS & 3800 & $<10$ & $<10$ & & \\
\hline
\end{tabular}

El tratamiento PRE-OSS logró en los trozos deshidratados (materia prima procesada) resultados variados en cuando al recuento de aerobios mesófilos viables, cuyos valores más altos estuvieron en los tratamientos $\mathrm{T} 3$ y $\mathrm{T} 7$, con $12 \times 10^{3}$ y $1 \times 10^{4} \mathrm{UFC} / \mathrm{g}$, respectivamente. También se logró resultados variados para el recuento de mohos, cuyos valores más elevados estuvieron en los tratamientos T2 y T5, con 110 y $70 \mathrm{UFC} / \mathrm{g}$, respectivamente. En relación al recuento de levaduras se observó que para casi todos los tratamientos el contenido fue $<10 \mathrm{UFC} / \mathrm{g}$, a excepción del T5 que se reportó $10 \mathrm{UFC} / \mathrm{g}$.

En la tabla 3 se presentan los resultados de la composición físico-químico del yacón en trozos deshidratados.

Se puede observar un alto contenido de carbohidratos, alcanzando un 93,2 \% en base seca. Al respecto, algunos autores ${ }^{7,15}$ reportaron que el contenido de carbohidratos totales del yacón varía entre 89,53 a 94,14 \% en base seca, confirmando así que estos nutrientes representan alrededor del $90 \%$ del peso seco del yacón ${ }^{8}$. 
Tabla 3. Análisis químico proximal, contenido de fructooligosacáridos y valor calórico del yacón en trozos antes del tratamiento OSS.

\begin{tabular}{lcc}
\hline \multirow{2}{*}{ COMPONENTES } & \multicolumn{2}{c}{ Trozos de yacón deshidratado } \\
\cline { 2 - 3 } & b.h. & b.s. \\
\hline Humedad $(\mathrm{g} / 100 \mathrm{~g})$ & 4,1 & - \\
Proteína $(\mathrm{g} / 100 \mathrm{~g})$ & 2,2 & 2,3 \\
Grasa $(\mathrm{g} / 100 \mathrm{~g})$ & 0,3 & 0,3 \\
Fibra $(\mathrm{g} / 100 \mathrm{~g})$ & 0,0 & 0,0 \\
Cenizas $(\mathrm{g} / 100 \mathrm{~g})$ & 4 & 4.2 \\
Carbohidratos $(\mathrm{g} / 100 \mathrm{~g})$ & 89,4 & 93,2 \\
Fructooligosacáridos $(\mathrm{g} / 100 \mathrm{~g})$ & 48,1 & 50,2 \\
Valor calórico (Kcal) & 369,1 & \\
\hline
\end{tabular}

Los trozos deshidratados del yacón presentaron un 50,2 \% de fructooligosacáridos. Investigaciones sobre el contenido de fructooligosacáridos (FOS) del yacón reportaron valores promedio en base seca que varían entre 59,61 a 78,3 \% ${ }^{9,11,15}$. En tal sentido, algunos investigadores mencionan que entre el 40 y $70 \%$ de los carbohidratos del yacón, son fructooligosacáridos (FOS) $)^{12}$, el resto lo conforman sacarosa, fructosa y glucosa ${ }^{11}$. Sin embargo, la composición relativa de los diferentes azúcares varía de acuerdo al cultivo, la época de siembra y cosecha, el tiempo y temperatura post cosecha.

\section{Descontaminación microbiana por el sistema de esterilización orgánica (Tratamiento OSS). \\ En la tabla 4 se muestra el comportamiento de la carga microbiana del yacón después del Tratamiento OSS, los factores evaluados fueron: Carga ( $\mathrm{kg} / \mathrm{saquito})$, tiempo (min) y temperatura $\left({ }^{\circ} \mathrm{C}\right)$.}

Tabla 4. Comportamiento de la carga microbiana del yacón después del tratamiento OSS.

\begin{tabular}{|c|c|c|c|c|c|c|c|c|c|c|}
\hline \multirow{2}{*}{ Tratsmientos } & \multirow{2}{*}{$\begin{array}{l}\text { Momento } \\
\text { del suslinis }\end{array}$} & \multirow{2}{*}{$\underset{\text { (ave iaguillo) }}{\text { Cara }}$} & \multirow{2}{*}{$\begin{array}{c}\text { Tiempo } \\
\text { de } \\
\text { proceso } \\
\text { (mia) }\end{array}$} & \multirow{2}{*}{$\begin{array}{c}\text { Temperatura } \\
\text { de inyeccióa } \\
\text { rapor } \\
\text { ("C) }\end{array}$} & \multicolumn{6}{|c|}{ Carga microbiana } \\
\hline & & & & & $\begin{array}{l}\text { Aerobios } \\
\text { (TFC E) }\end{array}$ & $\begin{array}{l}\text { Mohos } \\
\text { (CFCD) }\end{array}$ & $\begin{array}{c}\text { Levadaras } \\
\text { (CFC E) }\end{array}$ & 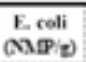 & $\begin{array}{l}\text { Salmosella } \\
\text { (TFC } \text { ) }\end{array}$ & $\begin{array}{c}\text { Colifermes } \\
\text { CXa/z) }\end{array}$ \\
\hline 1 & POST OSS & 3 & 1 & 100 & 10 & $<10$ & $<10$ & $<3$ & Ausencia & $<3$ \\
\hline 2 & POST OSS & 4 & 1 & 100 & 20 & $<10$ & $<10$ & $<3$ & Auseneia & $<3$ \\
\hline 3 & POST OSS & 3 & 2 & 100 & 70 & $<10$ & $<10$ & $<3$ & Ausencia & $<3$ \\
\hline 4 & POST OSS & 4 & 2 & 100 & 10 & $<10$ & $<10$ & $<3$ & Ausencia & $<$ \\
\hline 5 & POST OSS & 3 & 1 & 105 & $<10$ & $<10$ & $<10$ & $<3$ & Ausencia & $<3$ \\
\hline 6 & POST OSS & 4 & 1 & 105 & 20 & $<10$ & $<10$ & $<3$ & Ausencia & $<3$ \\
\hline 7 & POST OSS & 3 & 2 & 105 & 10 & $<10$ & $<10$ & $<3$ & Ausencia & $<3$ \\
\hline 8 & POST OSS & 4 & 2 & 105 & 10 & 10 & $<10$ & $<3$ & Ausencia & $<3$ \\
\hline
\end{tabular}


Las diferentes combinaciones de factores ( $\mathrm{kg} / \mathrm{saquillo}$, tiempo de proceso y temperatura de inyección de vapor) reportaron recuentos variados de aerobios entre $<10$ a $70 \mathrm{UFC} / \mathrm{g}$, mohos $<10$ a $10 \mathrm{UFC} / \mathrm{g}$ y levaduras $<10 \mathrm{UFC} / \mathrm{g}$. De los resultados obtenidos se determina que el tratamiento OSS ejerce acción sobre la carga microbiana.

Con el propósito de cuantificar la reducción de la carga microbiana se evaluó el Log (UFC/g) antes y después del tratamiento OSS (kg/saquillo, tiempo de proceso y temperatura de inyección de vapor saturado $\left({ }^{\circ} \mathrm{C}\right)$. En la tabla 5 se muestran los valores promedio de la reducción de aerobios, mohos y levaduras obtenidos: 80,47; 62,12 y 30,10\%; 60,59\%; $65,76 \%$ y $0 \%$, correspondientes a los tratamiento $5(3 \mathrm{~kg} /$ saquillo, tiempo de proceso de 1 minuto y $105^{\circ} \mathrm{C}$ de temperatura de vapor saturado $)$ y $2(4 \mathrm{~kg} / \mathrm{saquillo}$, tiempo de proceso de 1 minuto y $100{ }^{\circ} \mathrm{C}$ temperatura de vapor saturado), respectivamente. Asimismo, se encontraron valores muy similares, como: 73,$03 ; 58,8$ y $0 \%$ y $75 ; 46,28$ y $0 \%$, correspondientes a los tratamiento $4\left(4 \mathrm{~kg} /\right.$ saquillo, tiempo de proceso de 2 minutos y $100{ }^{\circ} \mathrm{C}$ temperatura de vapor saturado) y tramiento 7 ( $3 \mathrm{~kg}$ /saquillo, tiempo de proceso de 2 minutos y $105^{\circ} \mathrm{C}$ temperatura de vapor saturado), por lo que se puede inferir que el mejor comportamiento le correspondió al tratamiento 2, es decir con cargas de $4 \mathrm{~kg}$ /saquito, 1 minuto de tiempo de proceso y $100{ }^{\circ} \mathrm{C}$ de temperatura de inyección de vapor saturado.

Tabla 5. Reducción de la carga microbiana en función de Log (UFC/g) en yacón antes y después del tratamiento OSS.

\begin{tabular}{|c|c|c|c|c|c|c|c|c|c|c|}
\hline \multirow{2}{*}{ Tratsmiestos } & \multirow{2}{*}{$\begin{array}{l}\text { Momento } \\
\text { del snilisis }\end{array}$} & \multirow{2}{*}{$\begin{array}{c}\text { Carga } \\
\text { (ke/ iaquito) }\end{array}$} & \multirow{2}{*}{$\begin{array}{c}\text { Tiempo } \\
\text { de } \\
\text { proceso } \\
\text { (min) }\end{array}$} & \multirow{2}{*}{$\begin{array}{c}\text { Temperatura } \\
\text { de injeceib́n } \\
\text { vaper } \\
\text { ("C) }\end{array}$} & \multicolumn{6}{|c|}{ Reducción carta mierobiana (\%) } \\
\hline & & & & & Aerobist & Mbones & Levaduras & E. coli & Salmosella & Coliformes \\
\hline 1 & POST OSS & 3 & 1 & 100 & 70,72 & 58,86 & 0,00 & $<3$ & Ausencia & $<3$ \\
\hline 2 & POST OSS & 4 & 1 & 100 & 60,59 & 65,76 & 0,00 & $<3$ & Ausencia & $<3$ \\
\hline 3 & POST OSS & 3 & 2 & 100 & 54,77 & 0,00 & 0,00 & $<$ & Ausencia & $<3$ \\
\hline 4 & POST OSS & 4 & 2 & 100 & 73,03 & 58,86 & 0,00 & $<3$ & Ausencia & $<3$ \\
\hline 5 & POST OSS & 3 & 1 & 105 & 80,47 & 62,12 & 30,10 & $<3$ & Ausencia & $<3$ \\
\hline 6 & POST OSS & 4 & 1 & 105 & 64,91 & 30,10 & 0,00 & $<3$ & Ausencia & $<3$ \\
\hline 7 & POST OSS & 3 & 2 & 105 & 75,00 & 46,28 & 0,00 & $<3$ & Ausencia & $<3$ \\
\hline 8 & POST OSS & 4 & 2 & 105 & 72,07 & $-43,07$ & 0,00 & $<3$ & Ausencia & $<3$ \\
\hline
\end{tabular}

El análisis de varianza (tabla 6) evidencia que la temperatura de inyección de vapor saturado presenta diferencias estadísticas significativas para la reducción de la carga microbiana: aerobios, mohos y levaduras. Además, se puede observar que la variable $\mathrm{kg} / \mathrm{saquillo}$ es el factor con menor efecto significativo sobre la reducción de la carga microbiana. 
Tabla 6. Análisis de varianza para la reducción de aerobios totales, mohos y levaduras.

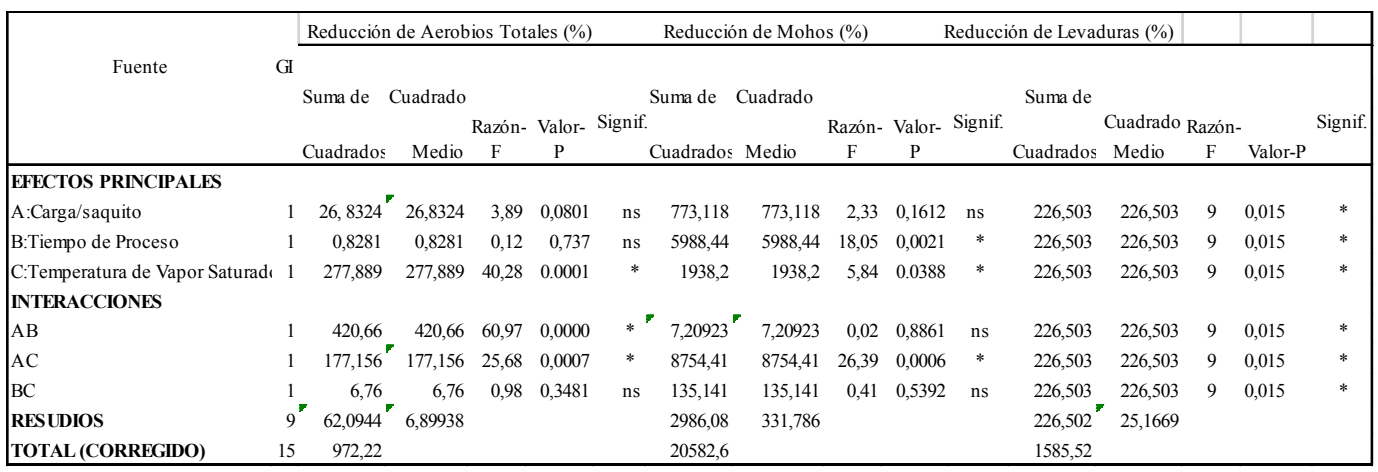

Del análisis de comparación de medias (Tukey), se concluye que no existe diferencia significativa entre los tratamientos. En tal sentido, teniendo en cuenta el análisis de varianza (ANVA), las variables y costos operativos del tratamiento OSS, permitieron determinar que el producto tratado con $4 \mathrm{~kg} /$ saquillo, 1 minuto de tiempo de proceso y con $100{ }^{\circ} \mathrm{C}$ de temperatura de inyección de vapor saturado (tratamiento 2), representa la combinación de factores con la máxima reducción de la carga microbiana: $10 \mathrm{UFC} / \mathrm{g}$ para aerobios $(60,59 \%)$, $20 \mathrm{UFC} / \mathrm{g}$ mohos $(65,76 \%) \mathrm{y}<10 \mathrm{UFC} / \mathrm{g}$ levaduras $(0 \%)$.

\section{Caracterización del yacón en polvo (producto final)}

\section{Caracterización química proximal y contenido de fructooligosacáridos del yacón en polvo.}

La tabla 7 presenta los resultados de las características físico-químicas del yacón en polvo, obtenidos con el tratamiento $2: 4 \mathrm{~kg} / \mathrm{saquillo}, 1$ minuto de tiempo de proceso y $100{ }^{\circ} \mathrm{C}$ de temperatura de inyección de vapor saturado.

El producto reportó una mayor humedad residual $(7,5 \%)$, con respecto a los trozos deshidratados (4,1\%, tratamiento PREOSS). Los contenidos de proteína y fructooligosacáridos no presentaron variación durante el tratamiento OSS, manteniéndose en 2,4 \% y $52 \%$ sobre la materia seca, respectivamente (tabla 7). Al respecto, investigaciones sobre el contenido de fructooligosacáridos del yacón en base seca, reportaron valores que variaron entre 44,15 a $57,11 \%{ }^{10,11,15}$. Este efecto se sustenta en que durante el tratamiento OSS el producto es expuesto a un ambiente saturado de vapor por un corto tiempo, que destruye los microorganismos de la superficie preservando al máximo las propiedades físicas y sensoriales. 
Tabla 7. Análisis químico proximal, contenido de fructooligosacáridos y valor calórico del yacón en polvo (mejor tratamiento).

\begin{tabular}{lcc}
\hline & \multicolumn{2}{c}{ Yacón en polvo } \\
\cline { 2 - 3 } COMPONENTES & b. h. & b.s. \\
\hline Humedad $(\mathrm{g} / 100 \mathrm{~g})$ & 7,5 & - \\
Proteína $(\mathrm{g} / 100 \mathrm{~g})$ & 2,2 & 2,4 \\
Grasa $(\mathrm{g} / 100 \mathrm{~g})$ & 0,0 & 0,0 \\
Fibra $(\mathrm{g} / 100 \mathrm{~g})$ & 2,2 & 2,4 \\
Cenizas $(\mathrm{g} / 100 \mathrm{~g})$ & 3,5 & 3,8 \\
Carbohidratos $(\mathrm{g} / 100 \mathrm{~g})$ & 86,8 & 93,8 \\
Fructooligosacáridos $(\mathrm{g} / 100 \mathrm{~g})$ & 48,1 & 52,0 \\
Valor calórico (Kcal) & 356 & \\
\hline \multicolumn{1}{c}{ b.h.=base húmeda } & & $b . s .=b a s e ~ s e c a$
\end{tabular}

\section{Análisis microbiológico del yacón en polvo}

En la tabla 8 se presentan los resultados del comportamiento de la carga microbiana en yacón en polvo, obtenidas en el mejor tratamiento.

Tabla 8. Comportamiento de la carga microbiana en yacón en polvo

\begin{tabular}{|c|c|c|c|c|c|c|c|c|c|}
\hline \multirow{2}{*}{ Tipo } & \multirow{2}{*}{$\begin{array}{c}\text { Carta } \\
\text { (kgessaquito) }\end{array}$} & \multirow{2}{*}{$\begin{array}{l}\text { Tiempo } \\
\text { (min) }\end{array}$} & \multirow{2}{*}{$\begin{array}{c}\text { Temperatura } \\
\text { de inyeccióa } \\
\text { vapor } \\
\text { ('C) }\end{array}$} & \multicolumn{6}{|c|}{ Carta mierobiana } \\
\hline & & & & $\begin{array}{l}\text { Aerobios } \\
\text { (UFC/g) }\end{array}$ & $\begin{array}{l}\text { Mobos } \\
\text { (UFC/g) }\end{array}$ & $\begin{array}{l}\text { Levaduras } \\
\text { (UFC g })\end{array}$ & 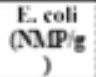 & $\begin{array}{l}\text { Salmonella } \\
\text { (UFC/E) }\end{array}$ & $\begin{array}{c}\text { Coliformes } \\
\text { (NMP/g) }\end{array}$ \\
\hline $\begin{array}{c}\text { Mlejor } \\
\text { Tratamiento } \\
\text { (T2) }\end{array}$ & 4 & 1 & 100 & 10 & 20 & $<10$ & $<3$ & ausencia & $<3$ \\
\hline
\end{tabular}

En la tabla 9 se presentan los estándares de calidad sanitaria peruana y requisitos microbiológicos internacionales para la exportación de productos a Japón y USA.

Tabla 9. Estándares de calidad sanitaria peruana y requisitos microbiológicos internacionales para la exportación de productos a Japón y USA33.

\begin{tabular}{|c|c|c|c|c|c|c|c|}
\hline \multirow{2}{*}{ Norma } & \multicolumn{5}{|c|}{ Recuento microbiológico } \\
\cline { 2 - 8 } & $\begin{array}{c}\text { Aerobios } \\
(\mathrm{UFC} / \mathrm{g})\end{array}$ & $\begin{array}{c}\text { Mohos } \\
(\mathrm{UFC} / \mathrm{g})\end{array}$ & $\begin{array}{c}\text { Levaduras } \\
(\mathrm{UFC} / \mathrm{g})\end{array}$ & $\begin{array}{c}\text { E. coli } \\
\text { (UFC/g) }\end{array}$ & $\begin{array}{c}\text { Salmonella } \\
\text { (UFC/g) }\end{array}$ & $\begin{array}{c}\text { Coliformes } \\
\text { (UFC/g) }\end{array}$ \\
\hline Sanitaria Peruana & & 100 & 100 & 10 & Ausencia/25g & $\ldots$ \\
\hline Internacional Japonesa & $<3000$ & $<300$ & $<300$ & Ausencia & Ausencia & Ausencia \\
\hline Internacional USA & $<10000$ & $<1000$ & $<1000$ & Ausencia & Ausencia & Ausencia \\
\hline
\end{tabular}


Los resultados, respecto a la presencia de carga microbiana del producto obtenido, cumplen con los requisitos microbiológicos establecidos por la legislación sanitaria peruana y las normas internacionales exigidas por Japón ${ }^{13}$ y USA ${ }^{14}$; por tanto, se puede afirmar que el tratamiento del producto con el Sistema de Esterilización Orgánica (OSS) logra la descontaminación microbiana del producto preservando al máximo las propiedades nutricionales y funcionales.

\section{CONCLUSIONES}

- Los parámetros del sistema de esterilización orgánica (OSS) que permitió la máxima reducción de carga microbiana del yacón fue con $4 \mathrm{~kg} / \mathrm{saquillo}, 1$ minuto de tiempo de proceso y $100{ }^{\circ} \mathrm{C}$ temperatura de inyección de vapor saturado.

- El yacón en polvo obtenido presentó una reducción de la carga microbiana: 60,59 \% (10 UFC/g) para aerobios, 65,76 \% (20 UFC/g) mohos y $0 \%(<10 \mathrm{UFC} / \mathrm{g})$ levaduras. El comportamiento de patógenos del yacón después del tratamiento OSS fue muy similar al de la materia prima: $E$. coli $<3 \mathrm{UFC} / \mathrm{g}$, ausencia de salmonella $(25 \mathrm{~g})$ y $<3 \mathrm{UFC} / \mathrm{g}$ para coliformes totales; indicando que el tratamiento aplicado logró la descontaminación microbiana a límites mínimos establecidos para el consumo humano, ajustándose a las exigencias de la norma sanitaria peruana y a las normas internacionales.

- Los contenidos de proteínas y fructooligosacáridos no sufrieron variación significativa durante el tratamiento OSS, obteniéndose $2,4 \%$ de proteína y $50 \%$ fructooligosacáridos (b.s.), logrando preservar la calidad nutricional y funcional del producto.

- La composición química del yacón en trozos, antes del tratamiento OSS en g/100g (b.s.) fue: Proteína 2,3; grasa 0,3; fibra 0,0; ceniza 4,2; carbohidratos 93,2 y fructooligosacáridos 50,2; y de la harina de yacón después del tratamiento con la tecnología OSS en g/100g (b.s.) fue: Proteína 2,4; grasa 0,0; fibra 2,4; ceniza 3,8; carbohidratos 93,8 , fructooligosacáridos 52,0.

\section{AGRADECIMIENTO}

Al Programa Nacional de Innovación para la Competitividad y Productividad (FIDECOM), por financiar esta investigación.

A la empresa Peruvian Nature S\&S SAC, propietaria del equipo OSS, donde se realizó la parte experimental de la investigación

\section{BIBLIOGRAFÍA}

1. Lachman J, Fernández EC, Orsák M. Yacon [Smallanthus sonchifolia (Poepp. et Endl.) H. Robinson] chemical composition and use-a review. Plant Soil Environ. 2003; 49 (6): 283-290.

2. Lock O, Rojas R. Química y Farmacología de Smallanthus sonchifolius (Poepp.) H. Rob. ("Yacón"). Rev Quím. 2005; 19(1): 31-35. 
3. Organic sterilization system. OSS, The natural way to treat your products [Internet]. Lima; Peruvian Nature; 2014. [Citado el 01 jul. 2014]. Disponible en: http://www. peruviannature.com/oss.html

4. AOAC. Official Methods of Analysis. 18th Ed. Gaithersburg, MD: AOAC International; 2005. Methods: 925.40, 981.10, 942.05, 978.10.

5. Jaime L, Martin-Cabrejas M, Mollá E, López-Andréu F, Esteban R. Effect of Storage on Fructan and Fructooligosaccharide of Onion (Allium cepa L.). J Agric Food Chem. 2001; 49: 982-988.

6. ICMSF. Internacional Commission on Microbiological Specification for Foods. Vol. 1. 3rd Ed. Zaragoza: editorial Acribia; 2000.

7. Ramos R, Arias G. Evaluación químico bromatológica de las variedades yurac llajum, qello llajum y yurac checche de smallanthus sonchifolius (poepp \& endl).h. robinson (yacón) procedente de Puno. Ciencia e Investigación. 2010; 13(2): 72-76.

8. Lizárraga L, Ortega R, Vargas W, Vidal A. Cultivo del yacón (Polimnia sonchifolia). En resúmenes Curso Pre-congreso - IX Congreso Internacional de cultivos andinos. Cuzco; 1997.

9. Vilhena S, Câmara F, Piza I. Lima G. Contenido de fructanos en raíces tuberosas de yacón (Polymnia sonchifolia). Sociedad Mexicana de Nutrición y Tecnología de Alimentos. Cienc Tecnol Aliment. 2003 4(1):35-40.

10. Nieto C. Estudios agronómicos y bromatológicos en Jicama (Polimia sonchifolia Poep et Endl). Arch Lat Nut. 1991; 41(2):213-221.

11. Mindani, C. Efecto de las condiciones de proceso en las características del yacón (Smallanthus sonchifolius Poepp. \& Endl.) liofilizado. [Tesis para optar el grado de Magister Scientiae en Tecnología de Alimentos]. Lima: Universidad Nacional Agraria La Molina; 2009.

12. Chivarry C. Influencia de las condiciones de Almacenaje del yacón fresco (Smallanthus sonchifolius) en sus compuestos bioactivos. [Tesis para optar el título de Ingeniero en Industrias Alimentarias]. Lima: Universidad Nacional Agraria la Molina; 2007.

13. MINCETUR. Guía de requisitos sanitarios y fitosanitarios para exportar alimentos a Japón. [Internet]. Lima: MINCETUR; 2010. [Accesado el 10 Jul 2014]. Disponible en: http://www.siicex.gob.pe/siicex/resources/calidad/req_japon.pdf.

14. MINCETUR. Guía de requisitos sanitarios y fitosanitarios para exportar alimentos a USA. [Internet]. Lima: MINCETUR; 2010. [Accesado el 10 Jul 2014]. Disponible en: http://www.siicex.gob.pe/siicex/resources/calidad/req_usa.pdf.

15. Cancino K, Guevara A. Influencia de la concentración del zumo en la deshidratación osmótica del yacón (Smallanthus sonchifolius Poepp. \& Endl.). Anales Científicos UNALM. 2006; 15(64): 225-243. 